04

\title{
Модель генерации нейтронов в малогабаритном диоде с лазерно-плазменным анодом и подавлением электронной проводимости постоянным цилиндрическим магнитом
}

\author{
(C) А.Е. Шиканов, Е.Д. Вовченко, К.И. Козловский, В.И. Ращиков, В.Л. Шатохин
}

Национальный исследовательский ядерный университет „МИФИ“, 115409 Москва, Россия

e-mail: vlshatokhin@mephi.ru

(Поступило в Редакцию 24 июля 2017 г.)

\begin{abstract}
Приведены результаты работ по созданию модели ускорения дейтронов и генерации нейтронов в малогабаритном лазерно-плазменном диоде с изоляцией электронов полем постоянного магнита, выполненного в виде полого цилиндра. Для рассматриваемой конструкции ускорительного диода сопоставлены результаты нейтронного выхода, полученные в эксперименте и при компьютерном моделировании. Показана возможность создания ускорительной нейтронной трубки с высоким энергетическим КПД генерации нейтронов за счет подавления электронной проводимости таким магнитом, расположенным в ее вакуумном объеме.
\end{abstract}

DOI: 10.21883/JTF.2018.04.45720.2445

\section{Введение}

Малогабаритные импульсные нейтронные генераторы на базе запаянных диодных ускорительных трубок (УТ) широко используются для нейтронного каротажа нефтегазовых и рудных скважин, экспрессного элементного анализа состава вещества, нейтронной томографии, а также обнаружения и идентификации скрытых опасных веществ и предметов [1].

Для повышения энергетического КПД генерации нейтронов в УТ используются различные способы подавления электронной эмиссии с катода диодной ускоряющей системы [2]. При большом ускоряющем напряжении и токах электростатические системы подавления электронной проводимости перестают быть эффективными. Поэтому было предложено для этой цели использовать принцип магнитной изоляции диодного промежутка [3].

В работах [4-6] было исследовано несколько вариантов реализации такого подхода. На основании результатов проведенных экспериментов и теоретических расчетов авторами был сделан вывод, что наиболее перспективной из рассмотренных является схема, использующая постоянный магнит с продольной намагниченностью, выполненный из материала на основе соединения $\mathrm{NdFeB}$ в виде полого цилиндра и расположенный внутри рабочего вакуумного объема УТ [5,6]. В работе [6] опубликованы первые результаты по генерации нейтронов на действующем макете такого диода с титановой мишенью, насыщенной дейтерием и бомбардируемой ускоренными дейтронами, из которых следует, что применение подобной магнитной изоляции в рассматриваемом биполярном диоде приводит к увеличению нейтронного выхода примерно на порядок.

Настоящая работа является логическим продолжением исследований, описанных в $[5,6]$. В ней осуществлено построение математической модели рассматриваемой диодной системы, базирующейся на результатах физического эксперимента. Моделирование позволило наметить пути модернизации запаянной конструкции ускорительного диода с магнитной изоляцией [3], пригодной для использования в малогабаритных эксплуатационных нейтронных генераторах и способной создавать высокие мгновенные потоки в полный телесный угол.

\section{Физическое моделироване диода}

На рис. 1 приводится схема экспериментальной установки для моделирования физических характеристик рассматриваемого диода, подробно описанная в [6].

Установка оснащена лазерным источником дейтронов [7] и генератором импульсного напряжения (ГИН), выполненным по схеме Аркадьева-Маркса с однополярной зарядкой [8]. ГИН состоит из 20 каскадов, в каждом из которых установлена цепочка из искрового разрядника, конденсатора емкостью $C=4700 \mathrm{pF}$ и зарядного сопротивления $R=16 \mathrm{k} \Omega$. При зарядном напряжении $U_{c} \approx 17.5 \mathrm{kV}$ в каждом каскаде запасается энергия $\sim 0.5 \mathrm{~J}$. В режиме холостого хода максимальная амплитуда выходного напряжения ГИН составляла величину $U_{m} \approx 350 \mathrm{kV}$, длительность фронта не более $0.1 \mu \mathrm{s}$. Давление в рабочем объеме составляло $\sim 5 \cdot 10^{-2} \mathrm{~Pa}$.

В диоде осуществлялось прямое ускорение к катоду дейтронов, извлекаемых из лазерной плазмы. Ускоряющее электрическое поле формировалось при подаче на анод импульса положительного высокого напряжения. На торце анода располагалась плазмообразующая мишень в виде таблетки из титана, насыщенного дейтерием на всю глубину с коэффициентом стехиометрии по дейтерию $s_{p} \approx 1.2$, на которую фокусировалось излучение твердотельного лазера с активным элементом 


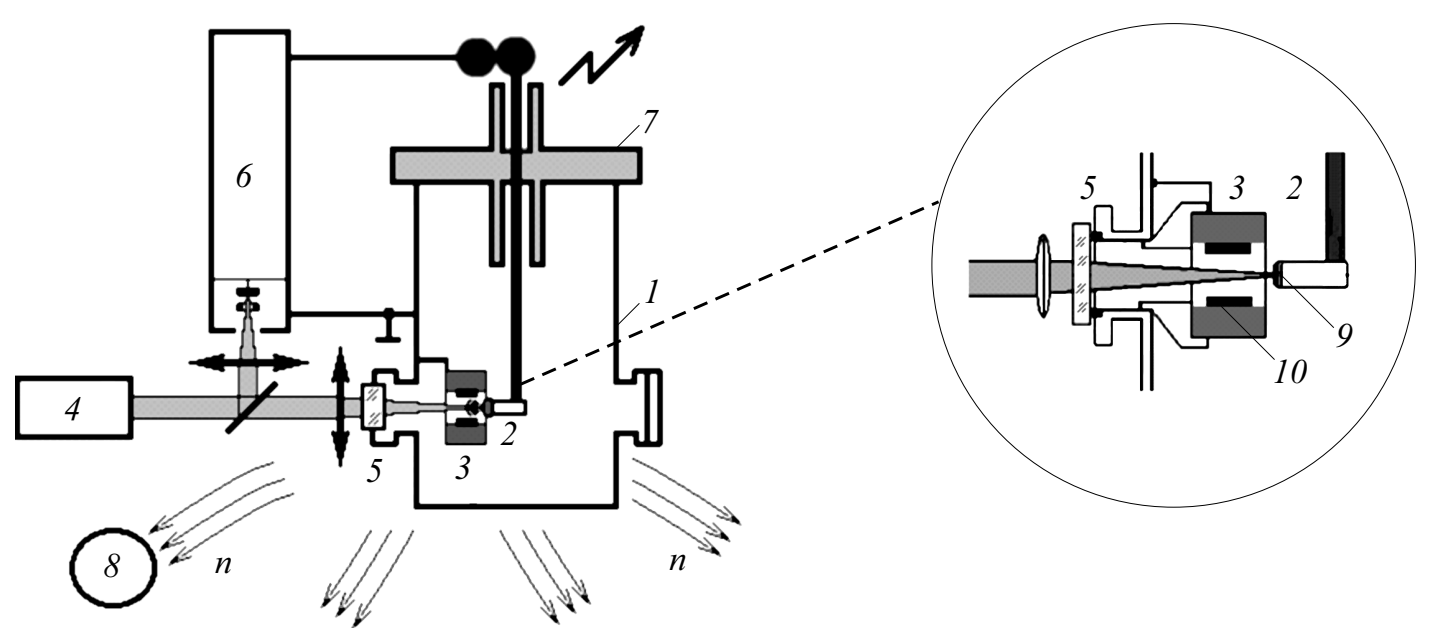

Рис. 1. Схема экспериментальной установки для исследования генерации нейтронов в плазменном диоде: $1-$ вакуумная камера, 2 - лазерно-плазменный анод, 3 - магнитный катод, 4 - импульсный лазер, 5 - оптическое окно, 6 - ГИН Аркадьева-Маркса, 7 - изолятор с электрическим вводом, 8 - детектор нейтронов, 9 - плазмообразующая мишень в виде таблетки TiD, 10 нейтроннообразующая мишень. На вставке - более крупно показана геометрия электродов.
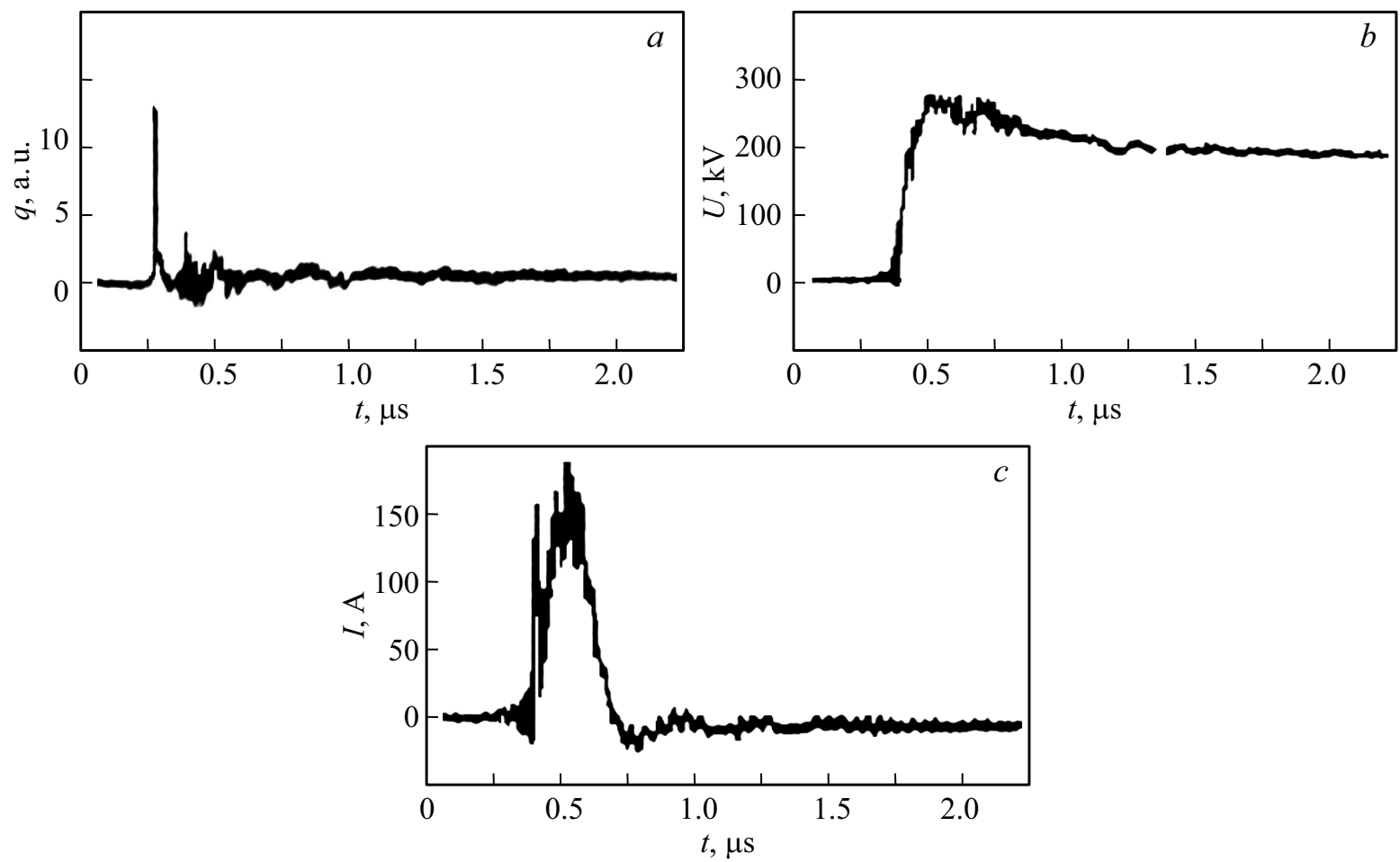

Рис. 2. Импульсы потока энергии лазерного излучения $(a)$, ускоряющего напряжения $(b)$, диодного тока $(c)$.

на иттрий-алюминиевом гранате, активированном неодимом с длиной волны $\lambda=1.06 \mu \mathrm{m}$. Плотность мощности лазерного излучения в области образования плазмы составляла $q \approx 5 \cdot 10^{10} \mathrm{~W} / \mathrm{cm}^{2}$, длительность лазерного импульса $\tau_{l} \approx 10 \mathrm{~ns}$.

Для синхронизации лазерного и ускоряющего импульсов выбрана „оптическая“ схема запуска ГИН, в которой часть лазерного импульса (до $25 \%$ от полной энергии пучка) направлялась на искровой разрядник в первом каскаде ГИН, вызывая его пробой и коммутацию в остальных секциях. При этом задержка импульса ускоряющего напряжения относительно лазерного импульса, как правило, не превышала 100-200 ns.

Катодный электрод выполнен из магнитного материала на основе соединений $\mathrm{NdFeB}$ в виде пустотелого цилиндра, тело которого образовано пересечением двух цилиндрических поверхностей: $r=R_{1,2}$ с плоскостями $z= \pm h / 2(r, z-$ цилиндрические координа- 
Результаты нейтронных измерений

\begin{tabular}{c|c|c|c|c}
\hline$U_{c}, \mathrm{kV}$ & $U_{0}, \mathrm{kV}$ & $\Delta Q, \mathrm{n} / \mathrm{pulse}$ & $Q, \mathrm{n} /$ pulse & $\delta, \%$ \\
\hline 7.5 & 120 & $5.30 \cdot 10^{4}$ & $1.01 \cdot 10^{6}$ & 27 \\
10 & 160 & $1.01 \cdot 10^{5}$ & $1.92 \cdot 10^{6}$ & 25 \\
12.5 & 200 & $2.49 \cdot 10^{5}$ & $4.74 \cdot 10^{6}$ & 25 \\
15 & 240 & $6.52 \cdot 10^{5}$ & $1.24 \cdot 10^{7}$ & 24 \\
17.5 & 280 & $2.50 \cdot 10^{6}$ & $4.75 \cdot 10^{7}$ & 24
\end{tabular}

ты), где $R_{1}=2.25 \mathrm{~cm}-$ внутренний радиус цилиндра, $R_{2}=4.25 \mathrm{~cm}$ - его внешний радиус, а $h=4 \mathrm{~cm}-$ высота.

На внутренней поверхности катода размещалась пробная нейтронообразующая мишень в виде тонкой металлической пластинки площади $\Delta S$ с напыленной на нее титановой пленкой, насыщенной дейтерием до коэффициента стехиометрии $s \approx 1.3$. Из соображений радиационной безопасности в качестве мишени вся внутренняя катодная поверхность в экспериментах не использовалась.

Продольная намагниченность катода до значений индукции на его торцах $\sim 0.5$ Т позволяла осуществлять, как показали эксперименты, магнитную изоляцию ускоряющего зазора.

В качестве иллюстрации на рис. 2 показаны осциллограммы импульсов потока энергии лазерного излучения $q(t)$, ускоряющего напряжения $U(t)$ и диодного тока $I(t)$.

Для регистрации нейтронов использовалась модификация всеволнового детектора [9] с гелиевым счетчиком тепловых нейтронов. При калибровке детектора использовался стандартный плутоний-бериллиевый ампульный источник нейтронов с потоком $10^{5} \mathrm{n} / \mathrm{s}$ в полный телесный угол. Результаты измерений нейтронного выхода представлены в таблице.

В таблице введены следующие обозначения: $U_{c}-$ напряжение зарядки конденсаторов ГИН, $U_{0}$ - измеренная максимальная амплитуда ускоряющего импульса при нагрузке на диодный промежуток с лазерной плазмой, $\Delta Q$ - измеренный нейтронный выход за импульс в полный телесный угол с пробной мишени,

$$
Q=\frac{2 \pi R_{2} h}{\Delta S} \Delta Q
$$

- нейтронный выход с мишени, расположенной на всей внутренней поверхности катода, который может быть получен в условиях данного эксперимента, $\delta$ - относительная погрешность измерений при доверительной вероятности 0.95 .

\section{Восстановление картины магнитного поля по данным измерений}

Магнитное поле в рабочем объеме диода может быть представлено в виде суперпозиции полей, создаваемых молекулярными токами $I_{1 ; 2, n}, \quad n=((1-N)-(N-1))$, протекающими по частичным кольцевым поверхностям

$$
\Delta_{n}^{1,2}=\left\{r=R_{1,2}, z \in\left[\frac{h n}{2 N}, \frac{h(n+1)}{2 N}\right]\right\},
$$

на которые разбиваются внутренняя и внешняя цилиндрические поверхности катода ( $N$ - число разбиений). Совокупность чисел $\left\{I_{1 ; 2, n}\right\}$ задает $2 N$-мерный вектор

$$
\mathbf{I}=\left\{I_{1,0}, I_{1,1}, \ldots, I_{1, N-1}, I_{2,0}, I_{2,1}, \ldots, I_{2, N-1}\right\},
$$

определяющий распределение вектора индукции магнитного поля в рабочем объеме диода:

$$
\begin{aligned}
\mathbf{B}(r, z, \mathbf{I}) \cong \mathbf{e}_{\mathbf{r}}\left\{\sum_{k=0}^{N-1}\left[I_{1, k} H_{1}(r, z, k)+I_{2, k} H_{2}(r, z, k)\right]\right\} \\
+\mathbf{e}_{\mathbf{z}}\left\{\sum_{k=0}^{N-1}\left[I_{1, k} F_{1}(r, z, k)+I_{2, k} F_{2}(r, z, k)\right]\right\}, \\
H_{1,2}(r, z, k)=G_{r}\left(r, R_{1,2}, z-\frac{2 h k+1}{4 N}\right) \\
+G_{r}\left(r, R_{1,2}, z-\frac{1-2 h k}{2 N}\right), \\
F_{1,2}(r, z, k)=G_{z}\left(r, R_{1,2}, z-\frac{2 h k+1}{4 N}\right) \\
+G_{z}\left(r, R_{1,2}, z-\frac{1-2 h k}{2 N}\right),
\end{aligned}
$$

$\mathbf{e}_{\mathbf{r}}, \mathbf{e}_{\mathbf{z}}$ - орты цилиндрической системы координат,

$$
\begin{aligned}
& G_{r}(r, a, z)= \frac{\mu_{0}}{2 \pi}\left\{E[f(r, a, z)] \frac{a^{2}+r^{2}+z^{2}}{(a-r)^{2}+z^{2}}\right. \\
&-K[f(r, a, z)]\} \frac{1}{\sqrt{(a+r)^{2}+z^{2}}}, \\
& G_{z}(r, a, z)= \frac{\mu_{0}}{2 \pi} \times\left\{E[f(r, a, z)] \frac{a^{2}-r^{2}-z^{2}}{(a-r)^{2}+z^{2}}\right. \\
&+K[f(r, a, z)]\} \frac{1}{\sqrt{(a+r)^{2}+z^{2}}}, \\
& f(r, a, z)=\frac{4 a r}{(a+r)^{2}+z^{2}},
\end{aligned}
$$

$K(f), E(f)$ - полные эллиптические интегралы 1-го и 2-го рода соответственно. Функции $G_{r}(r, a, z)$ и $G_{z}(r, a, z)$ определяют радиальную и продольную составляющие вектора индукции магнитного поля, создаваемого тонким кольцом радиуса $a$ с единичным током в точках с координатами $r, z$ [10].

Оценки показали, что вклад в формирование магнитного поля в объеме диода торцевых молекулярных токов и токов, создаваемых заряженными частицами, мал и может в расчетах не учитываться. 

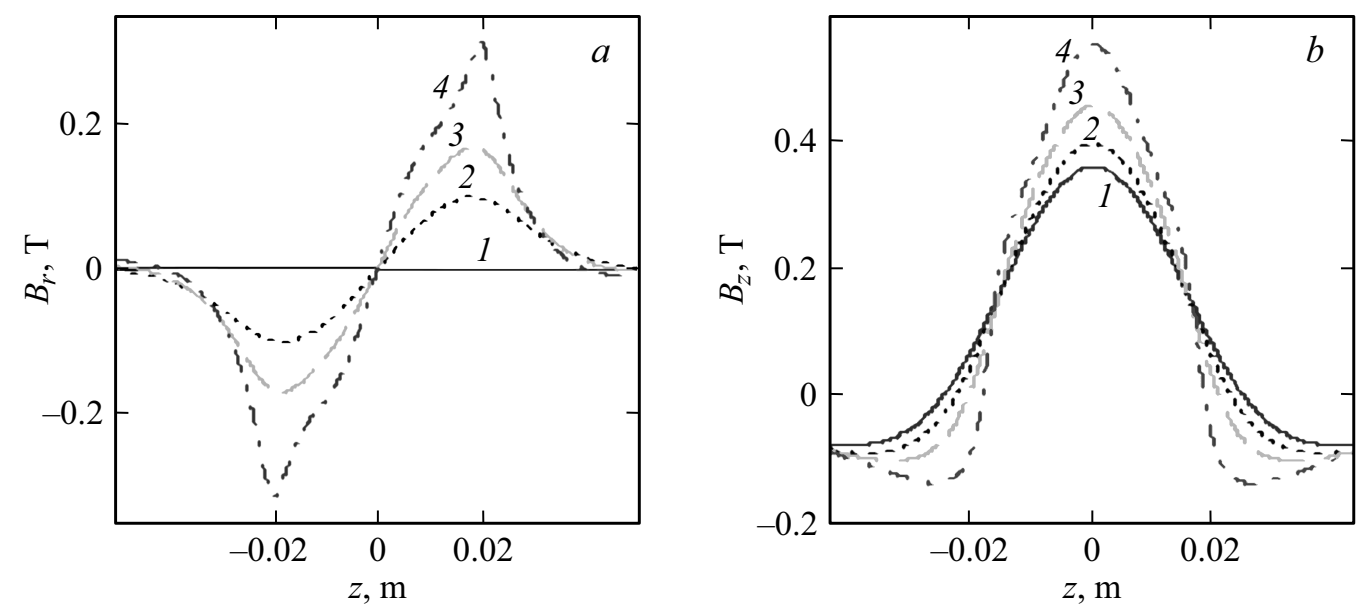

Рис. 3. Семейство зависимостей $B_{r}(r, z): 1-B_{r}(0, z), 2-B_{r}(0.010, z), 3-B_{r}(0.015, z), 4-B_{r}(0.020, z)-(a)$ и $B_{z}(r, z)$ : $1-B_{z}(0, z), 2-\tilde{B}_{z}(0.010, z), 3-B_{z}(0.015, z), 4-B_{z}(0.020, z)-(b)$.

Для определения коэффициентов $I_{1 ; 2, n}$ было проведено измерение продольной составляющей вектора индукции в трех точках с координатами $r_{1}=0, z_{1}=0, r_{2}=0$, $z_{2}=0.5 h$ и $r_{3}=0.5\left(R_{1}+R_{2}\right), z_{3}=0.5 h$. Им соответствуют измеренные значения продольной составляющей вектора индукции магнитного поля $\tilde{B}_{z 1}=0.36 T$, $\tilde{B}_{z 1}=0.125 T$ и $\tilde{B}_{z 3}=0.5 T$.

Нахождение искомых коэффициентов осуществлялось методом наименьших квадратов путем минимизации квадратичной формы

$$
W(\mathbf{I}, \gamma)=\sum_{i=1}^{3}\left[B_{z}\left(r_{i}, z_{i}, \mathbf{I}\right)-\tilde{B}_{z i}\right]^{2}+\gamma\|\mathbf{I}\|^{2},
$$

где $\gamma$ - параметр регуляризации Тихонова. Из условия $\frac{\partial}{\partial \mathbf{I}} W(I, \gamma)=0$ вытекает следующая система линейных алгебраических уравнений:

$$
\left\{\begin{array}{rl}
\sum_{k=0}^{N-1}\left[A_{1}^{1}(n, k)+\gamma \delta_{k, n}\right] I_{1, k} & +\sum_{k=0}^{N-1} A_{1}^{2}(n, k) I_{2, k} \\
& =\sum_{i=1}^{3} F_{1}\left(r_{i}, z_{i}, n\right) \tilde{B}_{z i}, \\
\sum_{k=0}^{N-1} A_{2}^{1}(n, k) I_{1, k}+\sum_{k=0}^{N-1}\left[A_{2}^{2}(n, k)+\gamma \delta_{k, n}\right] I_{2, k} & 8 \\
= & \sum_{i=1}^{8} F_{2}\left(r_{i}, z_{i}, n\right) \tilde{B}_{z i}
\end{array}\right.
$$

где

$$
\begin{aligned}
& A_{1}^{1}(n, k)=\sum_{i=1}^{3} F_{1}\left(r_{i}, z_{i}, n\right) F_{1}\left(r_{i}, z_{i}, k\right), \\
& A_{1}^{2}(n, k)=\sum_{i=1}^{3} F_{1}\left(r_{i}, z_{i}, n\right) F_{2}\left(r_{i}, z_{i}, k\right),
\end{aligned}
$$

$$
\begin{aligned}
& A_{2}^{1}(n, k)=\sum_{i=11}^{3} F_{2}\left(r_{i}, z_{i}, n\right) F_{1}\left(r_{i}, z_{i}, k\right), \\
& A_{2}^{2}(n, k)=\sum_{i=1}^{3} F_{2}\left(r_{i}, z_{i}, n\right) F_{2}\left(r_{i}, z_{i}, k\right) .
\end{aligned}
$$

На рис. 3 приводятся расчетные зависимости продольного и поперечного компонентов вектора индукции магнитного поля, используемые при компьютерном моделировании диода.

\section{Построение компьютерной модели диода}

Главной задачей настоящей работы являлось построение компьютерной модели рассматриваемого диода на основе анализа процессов формирования и ускорения в ней дейтронных и электронных потоков с использованием 2.5-мерного PIC (particle-in-cell) кода СУМА (Система уравнений Максвелла) [11,12]. Этот код осуществляет самосогласованный расчет динамики заряженных частиц в собственных и внешних электромагнитных полях методом „крупных частиц“.

Движение каждой крупной частицы описывается системой уравнений Ньютона-Лоренца:

$$
\left\{\begin{array}{l}
M_{\alpha} \frac{d \mathbf{V}_{\alpha}}{d t}=q_{\alpha} \mathbf{E}\left(r_{\alpha}, z_{\alpha}\right)+q_{\alpha}\left[\mathbf{V}_{\alpha}, \mathbf{B}\left(r_{\alpha}, z_{\alpha}\right)\right], \\
\frac{d \mathbf{r}_{\alpha}}{d t}=\mathbf{V}_{\alpha},
\end{array}\right.
$$

где $M_{\alpha}, Q_{\alpha}, r_{\alpha}, z_{\alpha}, \mathbf{V}_{\alpha}-$ соответственно масса, заряд, цилиндрические координаты и скорость частицы с номером $\alpha, \mathbf{E}\left(r_{\alpha}, z_{\alpha}\right)$ - напряженность электрического поля в точке нахождения частицы, $\mathbf{r}_{\alpha}=\mathbf{e}_{\mathbf{r}} r_{\alpha}+\mathbf{e}_{\mathbf{z}} z_{\alpha}-$ радиус-вектор точки нахождения частицы. Магнитное поле определялось в соответствии с формулой (1). 


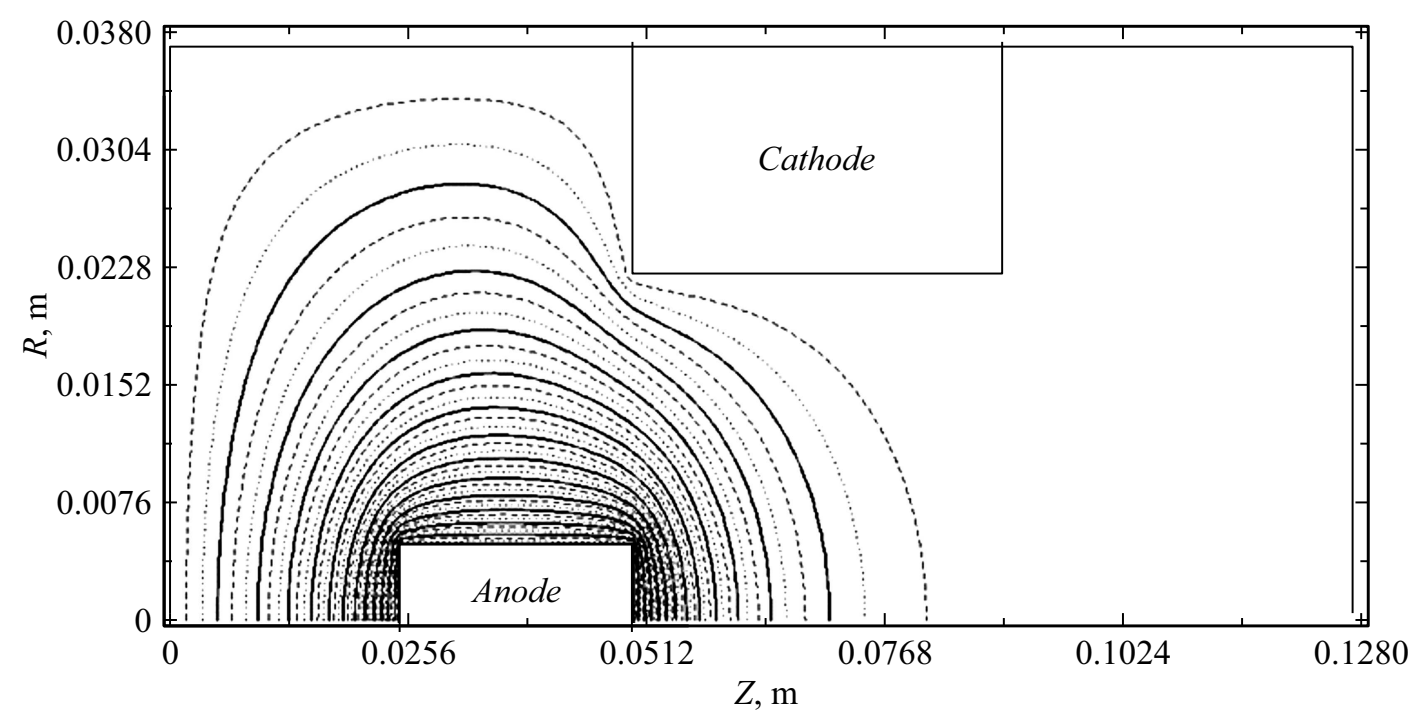

Рис. 4. Распределение потенциала в системе для $U_{0}=280 \mathrm{kV}$.

Для определения электрического поля на каждом шаге решения в текущий момент времени $t$ вычисляются плотности заряда и тока, входящие в уравнения Максвелла, осуществляется раздача заряда и токов в узлы пространственной сетки и их сглаживание путем взвешивания площадей (облака), соответствующих частице и ячейке сетки. Поступление новых частиц на шаге моделирования $\Delta t$ в рассматриваемую область реализовано в виде механизма инжекции с соответствующими законами распределения. После этого численно решаются уравнения Максвелла и осуществляется коррекция полученного решения на предмет соответствия уравнению Пуассона, так как часть частиц покидает область взаимодействия (уходит на мишень или другие граничные поверхности). Коррекция выполняется путем решения уравнения Пуассона для разности распределением плотностей заряда, полученным из дивергентного уравнения Максвелла, и реальным распределением зарядов $\rho(\mathbf{r})$ :

$$
\Delta \Phi_{c}(\mathbf{r})=\frac{\tilde{\rho}(\mathbf{r})-\rho(\mathbf{r})}{\varepsilon_{0}},
$$

где $\tilde{\rho}(\mathbf{r})=\varepsilon_{0} \operatorname{div} \mathbf{E}(\mathbf{r}), \Phi_{c}(\mathbf{r})-$ корректирующий потенциал, $\varepsilon_{0}$ - электрическая постоянная.

Скорректированное выражение для напряженности электрического поля имеет следующий вид:

$$
\mathbf{E}_{c}(\mathbf{r})=\mathbf{E}(\mathbf{r})-\operatorname{grad} \Phi_{c}(\mathbf{r}) .
$$

Уравнение Пуассона решается с использованием алгоритма быстрого преобразования Фурье по одной координате и прогонки (алгоритм Томаса) по второй. Для областей со сложной геометрией, а также при наличии электродов внутри области используется метод матрицы емкости, связывающий потенциал и заряд в необходимых узлах.

Поскольку в результате решения уравнений Максвелла поле находится в узлах пространственной сетки, для численного интегрирования уравнений движения необходимо вычисление поля в промежуточных точках, где располагаются частицы. Для этого используется интерполирование и сглаживание сеточных функций. Интегрируя уравнения движения (3), находим распределение частиц в фазовом пространстве в следующий момент времени: $t+\Delta t$ и т.д. Для интегрирования применен релятивистский вариант метода с перешагиванием с использованием временного сдвига пространственной координаты и импульса.

Основной проблемой решаемой задачи является корректное моделирование эмиссии дейтронов с поверхности разлетающейся в электромагнитном поле потока лазерной плазмы. В нашем случае в расчетах учитывается квазистатическое электрическое поле и поле постоянного магнита. Анализ экспериментальных данных [13,14], а также проведенные авторами коллекторные измерения позволяют оценить скорость разлета плазмы, соответствующую плотности потока лазерного излучения на плазмообразующей мишени $q \approx 5 \cdot 10^{10} \mathrm{~W} / \mathrm{cm}^{2}$, которая имела место в нашем случае. Так как для синхронизации высоковольтного импульса с временем образования лазерной плазмы, как уже говорилось выше, применялась схема с лазерным инициированием пробоя в разрядном промежутке первого каскада генератора Аркадьева-Маркса, задержка импульса ускоряющего напряжения по отношению к импульсу лазера составила 100 ns. Эти параметры, а также размер пятна лазера на плазмообразующей мишени позволяют оценить необходимые для моделирования исходные размеры границы плазмы к моменту появления в диоде импульса ускоряющего напряжения $U(t)$.

Граница потока плазмы к моменту прихода высоковольтного импульса представлялась в нашем случае цилиндрической поверхностью (рассматривался азимутально симметричный вариант) с исходными радиусом 


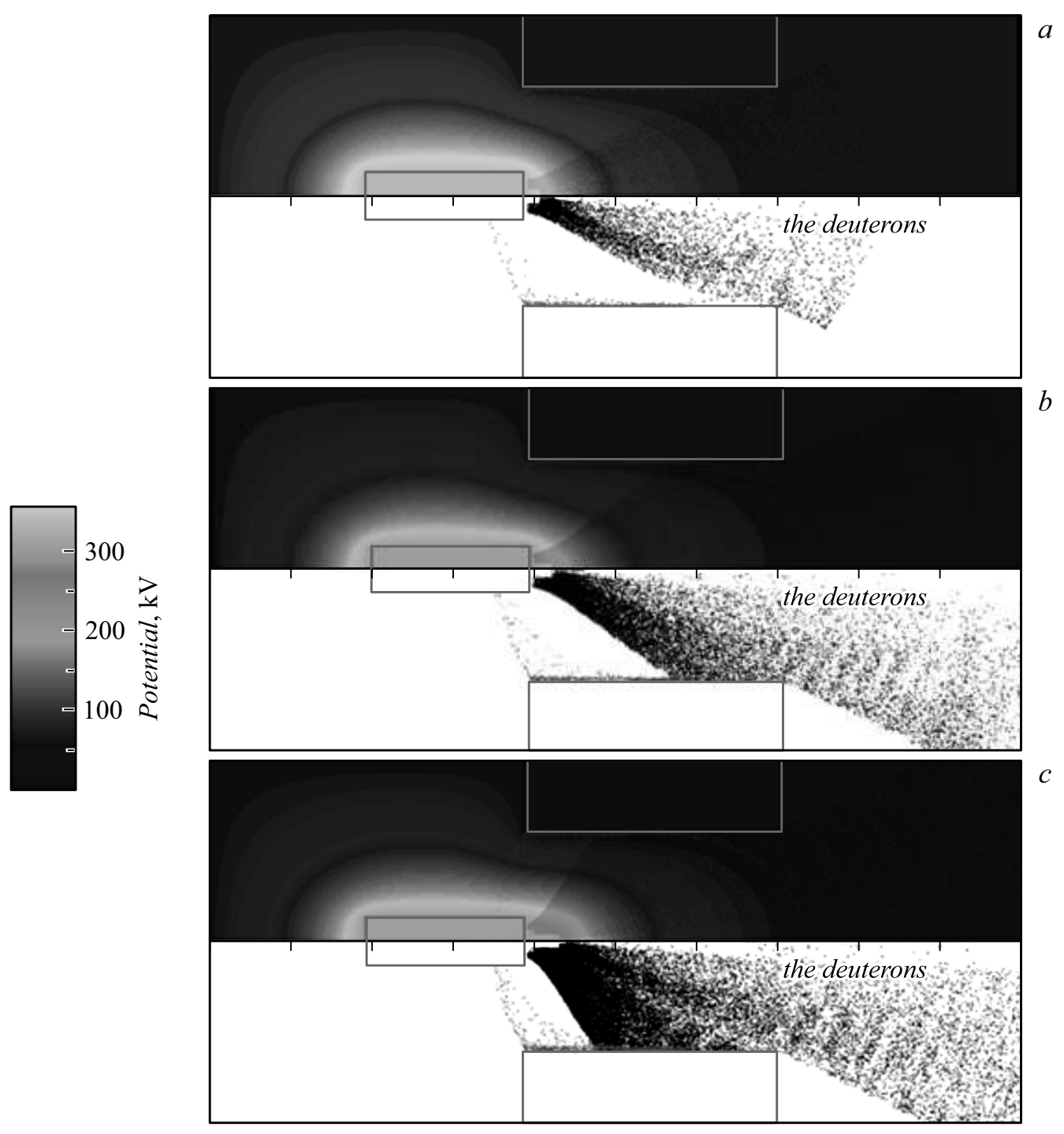

Рис. 5. Распределение потенциала (верхняя половина рисунка) и зарядов (нижняя половина рисунка) в диоде для различных моментов времени по отношению к лазерному импульсу: $a-150, b-200, c-250 \mathrm{~ns}$.

и длиной 1 и $2 \mathrm{~mm}$ соответственно. Затем она увеличивалась во времени в поперечном и продольном направлениях в соответствии с величиной соответствующей скорости. Эмиссия с границы плазмы моделировалась по дивергенции поля с учетом ограничения пространственным зарядом. Зависимость распределения электрического поля в системе от времени менялась в соответствии с изменением импульса ускоряющего напряжения $U(t)$ (рис. 2, $b$ ).

\section{Результаты компьютерного моделирования}

Распределение потенциала в диодной системе между кольцевым катодом и центральным анодом для фиксированного момента времени в отсутствие плазмы, представлено на рис. 4. В силу азимутальной сим- метрии показана только часть пространства выше оси системы.

Типичная картина динамики заряженных частиц и распределения потенциала электрического поля в системе в различные моменты времени представлена на рис. 5 . Электроны, отмеченные на рисунке точками на мишени и между левым краем мишени и анодом, появляются в диоде за счет вторичной эмиссии.

Полученные в процессе моделирования результаты для различных значений ускоряющего напряжения позволяют рассчитать нейтронный выход за импульс, излучаемый мишенью диода, по формуле [2]

$$
Q=\frac{s n}{e} \int_{0}^{\tau} d t I_{d}(t) \int_{0}^{e U_{c}(t)} d W \frac{\sigma(W)}{F(W)}
$$

где $s$ - коэффициент стехиометрии мишени, $n-$ концентрация ядер металла носителя мишени, $\tau-$ 


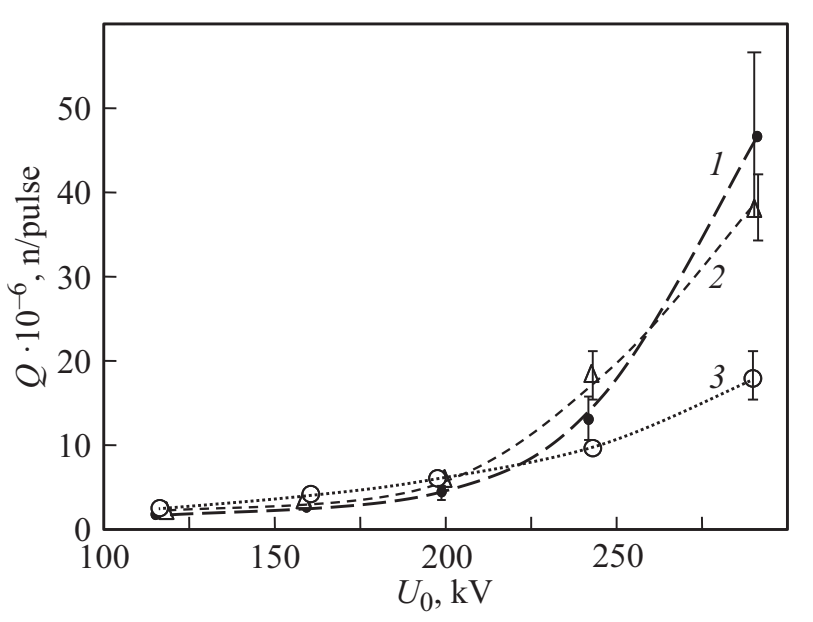

Рис. 6. Зависимость нейтронного выхода за импульс от ускоряющего напряжения на диодном промежутке: 1 - эксперимент (соответствует данным из вышеприведенной таблицы), 2 - уточненная компьютерная модель с учетом автоэлектронной эмиссии, 3 - первичная компьютерная модель. На кривых 2 и 3 показана погрешность, связанная с относительной точностью определения коэффициента стехиометрии $\sim 10 \%$.

длительность импульса дейтронного тока,

$$
\sigma(W)=\left(0.31+\frac{0.1-0.03 W^{2}}{W}\right) \exp \left(-\frac{1.4}{\sqrt{W}}\right)
$$

- микросечение ядерной реакции $D(d, n)^{3} \mathrm{He}$, barn;

$$
F(W)=\frac{n Z}{N_{A}} \sqrt{W}\left(\alpha \frac{1+\beta \sqrt{W}}{W^{1.275}+\gamma}+\frac{\alpha_{H}}{Z} \frac{1}{W^{1.275}+\gamma_{H}}\right)
$$

- тормозные потери дейтрона в мишени, $\mathrm{MeV} / \mathrm{m}$ [15], $Z$ - заряд ядер мишени, $N_{A}$ - число Авогадро. Расчет проводился для титановой мишени, насышенной дейтерием, с коэффициентом стехиометрии $s=1.3(\alpha=48$, $\left.\beta=0.078, \gamma=0.092, \alpha_{H}=136.9, \gamma_{H}=0.068\right)$. Длительность импульса составляла $\leq 0.25 \mu \mathrm{s}$.
Результаты реального компьютерного моделирования представлены кривой 3 на рис. 6 и достаточно хорошо совпадают с экспериментом в диапазоне амплитуд импульса ускоряющего напряжения 100-200 kV.

При более высоких напряжениях различия оказываются значительными. Анализ экспериментальных данных позволяет сделать предположение о том, что это отличие связано с появлением автоэлектронной эмиссии с левого края катодной нейтронообразующей мишени, где находится выступающая часть держателя магнита и где наибольшая напряженность электрического поля.

Автоэлектронная эмиссия может быть следствием ряда факторов, присутствующих в эксперименте. К ним относятся и наличие микроострий, связанное со степенью обработки поверхности мишени (магнита и его держателя), и возможное загрязнение поверхности. Кроме того, при больших амплитудах высоковольтного импульса в диоде появляется больше ионов титана, испаряемых с анода лазерным импульсом и вытягиваемых полем с поверхности плазмы. Чем больше напряжение на диоде, тем больше ионов титана достигают мишени, способствуя эмиссионным процессам. Распределение потенциала и зарядов в диоде с учетом автоэмиссии представлено на рис. 7.

Из сравнения рис. 5 и 7 видно, что появление дополнительной эмиссии с левого края мишени приводит к значительному изменению картины поля в диоде. Рост радиальной компоненты электрического поля на границе плазмы приводит к росту эмиссии дейтронов с ее поверхности и, как следствие, увеличению тока дейтронов на мишени. Этим фактом и вызвано дополнительное увеличение нейтронного потока. Уточненная компьютерная модель, учитывающая процессы автоэлектронной эмиссии в области усиления поля на краю катода, соответствует кривой 2 на рис. 6.

Анализ результатов моделирования показывает (рис. 5 и 7), что часть вторичных электронов с мишени попадает на анод. Иными словами, полной магнитной изоляции

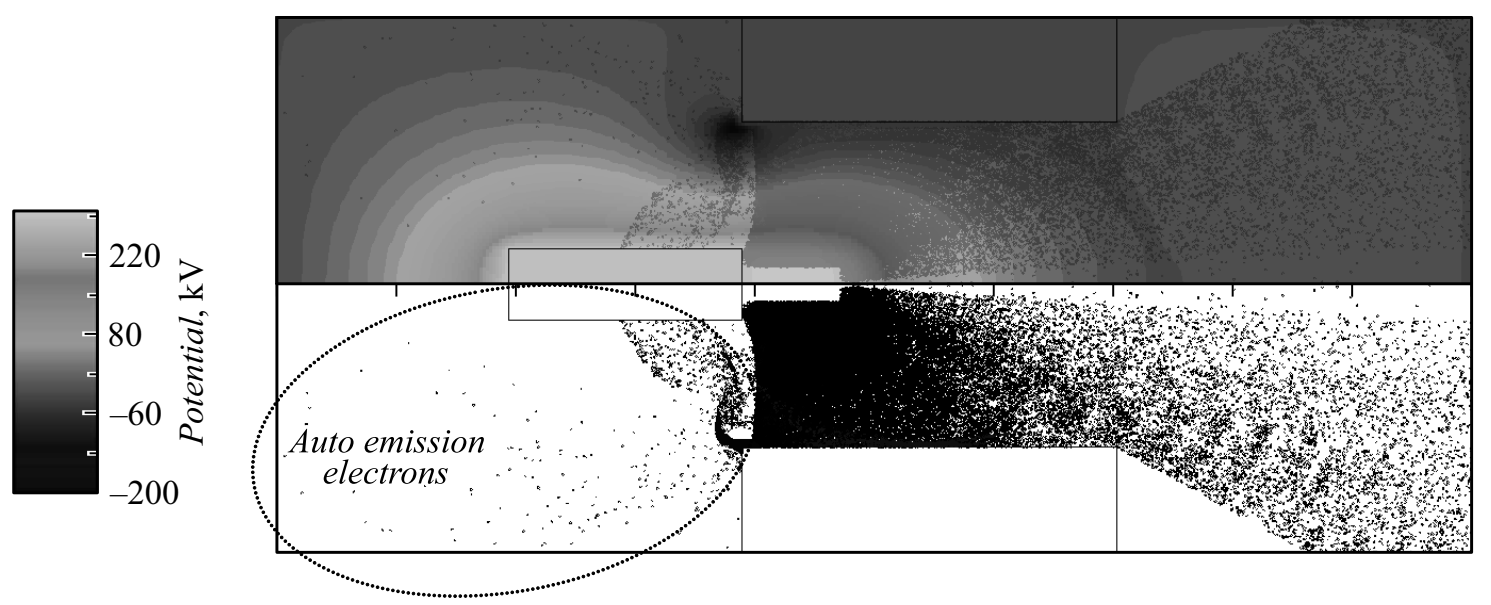

Рис. 7. Распределение потенциала (верхняя половина рисунка) и зарядов (нижняя половина рисунка) в диоде во времени с учетом автоэмиссии. Точки между левым краем мишени и анодом - электроны автоэмиссии. 


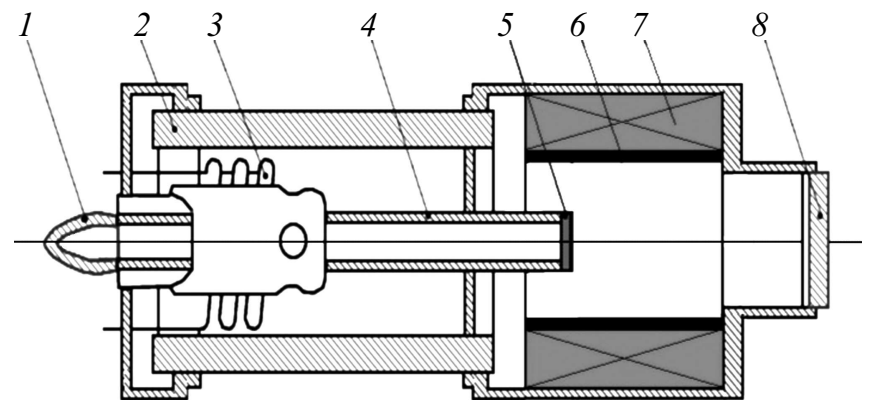

Рис. 8. Схематический разрез разрабатываемого варианта УТ с системой подавления электронной проводимости внутренним постоянным магнитом и лазерным источником дейтронов: 1 - штенгель для отпайки трубки, 2 - изолятор, 3 геттерный насос, 4 - анод, 5 - плазмообразующая мишень, 6 - нейтронообразующая мишень, $7-$ кольцевой магнит, 8 - окно для ввода лазерного излучения.

диода при данной величине и конфигурации магнитного поля не происходит. Расчеты дают величину такого тока при разных величинах амплитуды высоковольтного импульса в диапазоне 100-200 А, что достаточно хорошо согласуется с экспериментально измеренными токами диода (рис. 2,c). Величина же катодного тока оказывается в этом случае приблизительно в три-четыре раза большей.

По результатам физического и компьютерного моделирования авторами предложен возможный вариант реализации известной запаянной вакуумной УТ [3] (рис. 8) с мгновенным потоком нейтронов в полный телесный угол, существенно превышающим потоки, реализуемые на аналогичных изделиях, выпускаемых промышленностью или находящихся в стадии разработки.

\section{Заключение}

Проведенное моделирование показало достаточно хорошее совпадение полученных результатов с экспериментом в диапазоне амплитуд ускоряющего напряжения $120-280 \mathrm{kV}$. Экстраполяция полученных теоретических и экспериментальных данных по нейтронному выходу на более высокие напряжения говорит о возможности существенного роста нейтронного потока. Показано, что в диапазоне ускоряющего напряжения 200-280 kV на рост нейтронного выхода существенно влияют автоэмиссионные процессы на катоде. Планируются эксперименты по увеличению ускоряющего напряжения до $500 \mathrm{kV}$ и энергии импульса лазерного излучения до $1 \mathrm{~J}$, при которых ожидается повышение нейтронного выхода на порядок. Результаты моделирования показали, что увеличение эффективности генерации нейтронов возможно и за счет выбора более оптимальной конфигурации изолирующего магнитного поля в диоде.

Настоящая работа была выполнена в рамках повышения конкурентоспособности НИЯУ МИФИ.

\section{Список литературы}

[1] Сб. докладов Междунар. научно-техн. конф. „Портативные генераторы нейтронов и технологии на их основе“/ Под ред. Ю.Н. Бармакова. М.: ВНИИА, 2013. 620 с.

[2] Богданович Б.Ю., Нестерович А.В., Шиканов А.Е. Ворогушин М.Ф., Свистунов Ю.А. Дистанционный радиационный контроль с линейными ускорителями заряженных частиц. Т. 1. Линейные ускорители для генерации тормозного излучения и нейтронов. М.: Энергоатомиздат, 2009. $272 \mathrm{c}$.

[3] Беспалов Д.Ф., Козловский К.И., Цыбин А.С., Шиканов А.Е. Импульсная нейтронная трубка. А.c. СССР 766048. 1979.

[4] Диденко А.Н., Шиканов А.Е., Козловский К.И., Шатохин В.Л., Пономарев Д.Д. // Физика плазмы. 2014. Т. 40. № 11. C. $1025-1034$.

[5] Шиканов А.Е., Вовченко Е.Д., Козловский К.И., Шатохин В.Л. // Письма в ЖТФ. 2015. Т. 41. Вып. 10. C. $104-110$.

[6] Шиканов А.Е., Вовченко Е.Д., Козловский К.И. // Атомная энергия. 2015. Т. 119. Вып. 4. С. 210.

[7] Вергун И.И., Шиканов А.Е., Козловский К.И. и др. // ЖТФ. 1979. Т. 49. № 5. С. 2003-2006.

[8] Крастелев Е.Г., Лотоцкий А.П., Масленников С.П., Школьников Э.Я. Мощные электроимпульсные системы. Ч. II. Учебное пособие. М.: МИФИ, 2008. 144 с.

[9] Власов Н.А. Нейтроны. М.: Наука, 1971. 552 с.

[10] Ландау Л.Д., Лифиии, Е.М. Электродинамика сплошных сред. М.: Наука, 1982. 624 с.

[11] Ращиков В.И. // ВАНТ. Сер. Ядерно-физические исследования. 1990. Вып. 10(18). С. 50-53.

[12] Диденко А.Н., Ращиков В.И., Рыюкков В.И., Цыбин А.С., Шиканов А.Е. // Атомная энергия. 2012. Т. 112. Вып. 3. C. $182-184$.

[13] Козловский К.И., Козырев Ю.П., Цыбин А.С. // Физика плазмы. 1980. Т. 6. Вып. 1. С. 69-72.

[14] Быковский Ю.А., Васильев Н.М., Дегтяренко Н.Н., Елесин В.Ф., Лаптев И.Д., Неволин В.Н. // Письма в ЖЭТФ. 1972. Т. 15. Вып. 6. С. 308-311.

[15] Богданович Б.Ю., Нестерович А.В., Шиканов А.Е. // Атомная энергия. 2010. Т. 109. Вып. 5. С. 293-298. 